\title{
Transcriptome and metabolome analyses revealing the potential mechanism of seed germination in Polygonatum cyrtonema
}

\section{Rong Liu}

University of the Chinese Academy of Sciences

Jing Lu

University of the Chinese Academy of Sciences

Jiayi Xing

University of the Chinese Academy of Sciences

Chihong Zhang

Chengdu Institute of Biology, Chinese Academy of Sciences

\section{Min Zhou}

University of the Chinese Academy of Sciences

Mei Du

University of the Chinese Academy of Sciences

\section{Mingxiu Wang}

University of the Chinese Academy of Sciences

\section{Yunfang Li}

Chengdu Institute of Biology, Chinese Academy of Sciences

\section{Lei Zhang}

Chengdu Institute of Biology, Chinese Academy of Sciences

Yu Wu (D wuyu@cib.ac.cn )

Chengdu Institute of Biology, Chinese Academy of Sciences

\section{Research article}

Keywords: Polygonatum cyrtonema, transcriptomic, metabolomics, seed germination, RNA_seq, LC/MS

Posted Date: March 31st, 2020

DOI: https://doi.org/10.21203/rs.3.rs-19880/v1

License: (1) (1) This work is licensed under a Creative Commons Attribution 4.0 International License.

Read Full License 
Version of Record: A version of this preprint was published at Scientific Reports on June 9th, 2021. See the published version at https://doi.org/10.1038/s41598-021-91598-1. 


\section{Abstract}

Background: Polygonatum cyrtonema Hua is a Chinese traditional medicine in the Liliaceae family. It have unique medical and edible value. However, the Polygonatum cyrtonema seeds are naturally difficult to germinate. Its reproductive and growth cycles should be accelerated through artificial technologies for meeting the market demand. Therefore, to beak the seeds dormancy and to elucidate the mechanism of germination, the metabolomics and transcriptomics analysis were performed in this study.

Results: The results indicate that plant hormone (auxin, GA, ABA) were involved in seed germination. Furthermore, the expression levels of unigenes and a-amylase activity were increased in germinated seeds. In addition, the metabolites of phenylpropanoid and flavonoid biosynthesis were accumulated in Polygonatum cyrtonema seeds. Hhydroxycinnamyl (caffeic acid, coniferin and sinapic acid et al.), organic acids (benzoic acid, ferulic acid, and coumaric acid etc.) were significantly decreased, while flavonoids significantly accumulated during the germination. The results suggest that the metabolites of germinated seeds were mainly to promote flavonoids synthesis and to inhibit the lignin synthesis, which was beneficial to germination of Polygonatum cyrtonema. And hydrolysis of starch to glucose can provides necessary energy for germination.

Conclusions: Our results give a novel insights into the regulating networks of seed germination and pave the way for Polygonatum cyrtonema seeds propagation and cultivation.

\section{Background}

Polygonatum cyrtonema is a species in the family Liliaceae, commonly used in traditional Chinese medicine. It is known as Huangjing that widely grown in China. For its unique medical and edible value, Huangjing has been used for more than 2000 years in China. Huangjing has also been applied in therapy for weakness, such as anti-inflammatory, antimicrobial, hypoglycemic, and immunoregulatory[1].

Previous studies of Huangjing have primarily focused on the resource survey and cultivation [2], rhizome tissue culture[3], characterizing chemical composition[4] and drug effects [5]. However, little information about Huangjing seeds germination $(G)$. Huangjing seeds are naturally difficult to germinate, which will take more than 6 months of dormancy to germinate under natural sowing conditions. In order to meet with the rapid growth of the cultivation market demands of Huangjing in China. Therefore, the regulatory mechanism of the seeds germination should be comprehensive understanding to resolve reproductive problems and to accelerate growth cycles of Huangjing via artificial technologies.

Seed germination is tightly regulated from transcriptional and metabolite changes. Increasing information from transcriptomics and metabolomics studies has enhanced global understanding of the germination process and regulation. Metabolomics has recently become a major research tool to analyze substrates and products in metabolic pathways[6]. Many researches also attempts to developed the variation of gene expression and build comprehensive models of seed germination[7]. A few studies regarding metabolism during seed germination were conducted in Arabidopsis[8], barley[9] and rice[10]. In 
Polygonatum cyrtonema, non-study have focused on the metabolite and transcriptomic profiling of seed germination.

Germination is regulated by multiple endogenous factors, such as plant hormones, and by environmental conditions[11]. The phytohormones gibberellin acid (GA) and abscisic acid (ABA) play crucial roles in the regulation of seed germination by stimulating or suppressing seed germination[12]. In Polygonatum cyrtonema, carbohydrates and proteins stored in the endosperm are mobilized during seed germination to provide energy and substrates for developing seedlings[13]. GAs were synthesized to induce the gene expression and synthesis of a-amylase during seed germination[14-15]. Then, $a$-amylase was secreted into the endosperm to decomposed stored starch of seed[16]. At the same time, organic acids and flavonoids may participate in acidifying the endosperm tissue, releasing stored starch into metabolism to affect seed germination[17-19].

However, metabolomic and transcriptomic profiling during Huangjing seed germination remains unknown. To elucidate the mechanism of seeds germination, we performed the first integrated metabolomics and transcriptomics analysis of the mechanism of Huangjing seed germination using liquid chromatography-MS (LC/MS) and next generation sequencing (NGS). The unigenes expression or metabolites of starch and sucrose metabolism, flavonoid biosynthesis, plant hormone transduction, phenylpropanoid metabolism and a-amylase activity were investigated in this study. Our results provide novel insights into the biochemical mechanisms of Polygonatum cyrtonema seed germination at the metabolome and transcriptome level.

\section{Results}

Morphological identification and phenotype of Polygonatum cyrtonema

The plant morphology, flowers and tubers of Polygonatum cyrtonema Hua show in Fig. 1A, B, C. Figure 1B and Fig. 1C obtained from Plant Photo Bank of China, PPBC (http://ppbc.iplant.cn/). Polygonatum cyrtonema Hua is a perennial herb of the Liliaceae family, with thick rhizomes, alternate phyllotaxy and yellow-green perianth, flowering from May to June and fruiting from August to October. The medicinal part of Polygonatum is rhizome. In this study, the fruits and seeds (Fig. 1D, E) of Polygonatum cyrtonema Hua obtained from Chengdu Institute of Biology, Chinese Academy of Sciences. Germinated seeds (Fig. 1F) were collected for transcriptome sequencing and metabolome analysis after six months of planting.

RNA-seq de novo assembly and function annotation of Polygonatum cyrtonema

Six cDNA libraries, each for Huangjing seeds with germinated and control ( $G$ and CK) group, were characterized by Illumina HiSeq to detect the transcriptome level of gene expression information. A summary of RNA-Seq data is shown in Table 1, high-quality and clean reads were obtained by removing low-quality reads in per library. The Q30 of six CDNA libraries were more than $95 \%$ and GC was $52.33 \%$. After de novo assembly, a total of 56,628 unigenes (UG) were detected. Among them, there were 29,688 
(52.43\%) annotated in NR, 27,901 (49.27\%) were characterized from eggNOG database, 22,673 (40.04\%) annotated in SwissProt, 11,611 (20.50\%) annotated in KEGG, 20,543 (36.28\%) annotated in GO and annotated in KOG were 17,965 (31.72\%). A total of 33,705 unigenes > 500 bp and 15,223 unigenes > $1000 \mathrm{bp}$. The transcriptome assembly results revealed that the RNA-Seq datasets were reliable to further study.

Table 1

Statistics and functional annotations of unigenes in 6 RNA sequencing libraries

\begin{tabular}{|lll|}
\hline & Number of unigenes & Percentage (\%) \\
\hline$>300 \mathrm{bp}$ & 56628 & \\
>=500 bp & 33705 & 59.5 \\
>=1000 bp & 15223 & 26.9 \\
\hline N50 & $1127(\mathrm{bp})$ & - \\
\hline Max length & $11337(\mathrm{bp})$ & - \\
\hline Min length & $301(\mathrm{bp})$ & - \\
\hline Average length & $855(\mathrm{bp})$ & - \\
\hline NR & 29688 & 52.4 \\
\hline EggNOG & 27901 & 49.3 \\
\hline SwissProt & 22673 & 40.1 \\
\hline KEGG & 11611 & 20.5 \\
\hline GO & 20543 & 36.3 \\
\hline KOG & 17965 & 31.7 \\
\hline Total & 56628 & \\
\hline
\end{tabular}

Comparative Analysis And Qrt-pcr Verification

To elucidate the molecular basis for Huangjing seed germination, comparative transcriptome analysis was conducted. DEGs were analyzed using the RPKM method between the $G$ and CK group. Compared with the CK, a total of 18,308 DEGs up-regulated and 9,709 DEGs down-regulated in the sprouted Huangjing seeds (Fig. 2A, Supplemental File 1-1). The results indicating that these 27,017 DEGs might be involved in Huangjing seed germination.

To evaluate the accuracy of transcriptome profiles from the RNA sequencing analysis, 18 genes related of seed germination were selected for qRT-PCR expression analysis. The correlation of RNA-Seq (FPKM) and 
qRT-PCR are shown in Fig. 2B (Supplemental File 1-2). The relative gene expression level of qRT-PCR were consistent with RNA-Seq profile, which indicated that the RNA-Seq data were reliable.

Kegg Analysis Of Differentially Expressed Unigenes (degs)

To identify the major functional terms and signal pathways between $\mathrm{G}$ and CK seeds, KEGG analysis was carried out of DEGs at a significance level of $p<0.05$. The KEGG annotations indicated that plant hormone signal transduction (ko04075), phenylpropanoid biosynthesis (ko00940), starch and sucrose metabolism (ko00500),phenylalanine metabolism (ko00360), flavonoid biosynthesis (Ko00941) pathways were significantly enrichment in Huangjing seeds (Fig. 3, Supplemental File 2).

DEGs of phenylpropanoid and flavonoid biosynthesis related to seed germination

The DEGs involved in phenylpropanoid biosynthesis (ko00940) and flavonoid biosynthesis (Ko00941) were examined in this study, which involved in seed germination. A total of 114 DEGs (91 up-regulated, 23 down-regulated) were detect in phenylpropanoid biosynthesis, including PAL, CYP73A, CYP73A, 4CL and CCR, etc. Twenty-two DEGs were enriched in flavonoid biosynthesis and mostly up-regulated, such as CHS, FLS, F3Hand CHI et al. (Fig. 4A, Supplemental File 3). To validate the transcriptome expression data for genes involved in flavonoid biosynthesis, we randomly selected six genes for qPCR analysis. Including CHS (TRINITY_DN14701_c0_g2_i1_4, TRINITY_DN27324_c0_g1_i1_3), E5.5.1.6/Chalcone isomerase (TRINITY_DN39749_c0_g1_i5_3), F3H (TRINITY_DN16505_c0_g2_i1_4, TRINITY_DN16337_c0_g1_i1_4), and FLS (TRINITY_DN43663_c0_g1_i1_3). The fold changes of RNA_seq were all consistent with those from the qPCR analysis (Fig. 4B). Those results indicate that phenylpropanoid and flavonoid biosynthesis pathway are important for seed germination.

DEGs related to starch and sucrose metabolism in seed germination

The significant enriched pathway was found in G vs CK seeds, a total of 59 DEGs were detect in starch and sucrose metabolism (ko00500), 41 up- and 18 down-regulated (Supplemental File 4) in Huangjing. We found that a-amylase synthesize gene and MGAM enzyme genes significantly up-regulated during seed germination (Fig. 5A). Therefore, we determined the a-amylase activity and related gene expression in this pathway. The results show that the highest a-amylase activity was found in germination seeds, followed by the CK seeds and radicle (Fig. 5B). The enzyme genes of a-amylase, E2.4.1.15, E3.2.1.20, E3.2.1.39 and E3.2.1.21 in starch and sucrose metabolism were determined by qRT-PCR (Fig. 5C) and all DEGs were significantly up-regulated in germination seeds. The expression levels of unigenes were consistent with the trends of a-amylase activity, indicating that the activity of a-amylase and sucrose metabolism might play an important roles in promoting seed germination.

Plant Hormone Related Degs In Seed Germination

Plant hormone affect the germination of plant seeds, thus we found that plant hormone signal transduction (ko04075) were significantly enriched in this study. DEGs involved in the auxin synthesis regulation pathway, such as AUX1 and TIR1 were significantly up-regulated in germinated seeds (Fig. 6A, 
Supplemental File 5). The GID2 and TF in GA related pathway were also up-regulated. However, compare with $\mathrm{CK}$, the DEGs were almost down-regulated in ABA signal pathway, including PP2C, SnRK2 and ABF et al. In addition, we found that the DEGs were significantly changed in the upstream of salicylic acid synthesis (k000360, ko00362), while some genes in the downstream of salicylic acid pathway were also significantly different, such as NPR1, PR1. To validate the transcriptome expression data, three genes of plant hormone signal transduction, AUX1 (TRINITY_DN27428_c0_g1_i1_3), AUX/IAA

(TRINITY_DN32072_c0_g1_i1_2) and A-ARR (TRINITY_DN21518_c0_g1_i1_2) were randomly selected for qPCR analysis (Fig. 6B). The fold changes for the qPCR analysis indicated the relative expression of the germinated and the control seeds, and the fold changes of RNA_seq were consist with the GPCR analysis. Therefore, we suggest that auxin, GA and ABA were involved in seed germination.

Metabolic Analysis And Association Of Transcriptomic Analysis

To verify the accuracy of transcriptome sequencing results, we conducted metabolic analysis. In this study, a total of 637 metabolites were detected in Huangjing seeds by LC-MS, including certain primary and secondary metabolites, such as organic acids, amino acids, flavonoids, lipids etc. Compared with CK, 230 measured different metabolites ( 96 up-regulated and 134 down-regulated) were significantly to be identified in germination seeds (Fig. 7, Supplemental File 6). To study the association between transcriptomic and metabolic in seeds, we analyzed the connection of DEGs expression and metabolites. Certain key enzyme encoding DEGs were related to produce metabolites, this result reveal that these enzyme encoding DEGs might be involved in seeds germination.

Flavonoids And Hydroxycinnamyl Related To Seed Germination

We conducted a detailed analysis of the different metabolites in G vs CK seeds. The result shown that 42 flavonoids significantly accumulated during the germination of seeds (Fig. 8A, Supplemental File 7 - 1). Compare with CK, All 42 flavonoids were significantly increased in G group, including flavanone, flavonol and anthocyanins, etc. The result is consistent with the RNA_seq results. Therefore, we suggesting that the synthesis of flavonoids may play an important roles to the germination of Huangjing seeds.

In addition, we found that hydroxycinnamyl showed a significant down-regulated trend (Fig. 8B, Supplemental File 7 -2). Hydroxycinnamyl are precursors in the lignin synthesis, and the metabolites in this pathway may negatively regulate the germination of seeds. Therefore, we further analyzed the flavonoid synthesis and the lignin synthesis pathway, and found that both pathways are derived from the phenylalanine metabolism pathway. Based on the KEGG pathway, we mapped metabolic pathways related to germination of Huangjing. The metabolites of flavonoid synthesis pathway naringenin chlcone, naringenin, aromadedrin, taxifolin and cyaniding were significantly increased in germinated seeds. However, compared with $\mathrm{CK}$, the metabolites cinnamic acid, caffeic acid, caffeic aldehyde, coniferyl aldehyde, coniferin and sinapic acid were significantly decreased in phenylpropanoid pathway (Fig. 8C, Supplemental File 6). The results showed that the metabolites flow of phenylalanine pathway was mainly to promote flavonoids synthesis and to inhibit the lignin synthesis during the seeds germination, which was beneficial to the germination of Polygonatum cyrtonema seeds. 
Organic acids related to germination of Polygonatum cyrtonema

In present study, we found that organic acids significant down-regulate with the germination of Polygonatum cyrtonema seeds (Fig. 9, Supplemental File7-3). The results showed that organic acids could significantly inhibit seed germination. We further analyzed and found that benzoic acid, ferulic acid, and coumaric acid were the most significant organic acid metabolites during germination. Therefore, we speculated that these metabolites have a major inhibitory effect on the germination of Polygonatum cyrtonema seeds.

\section{Discussion}

The seed is a critical stage of agriculture and biodiversity for plant. Seed germination, an adaptive trait, is a crucial phases of the most important decision points in plant life cycle which is strongly related to seedling survival rate and subsequent growth of a higher plant [20-21]. Germination commences with the uptake of water and ends with visible radicle emergence through the seed coat[22]. According to Baskin et al.[23] the criteria for the classification of seed dormancy, Polygonatum cyrtonema belongs to morphological and physiological dormancy.

\section{Flavonoid Synthesis Associated With Seed Germination}

To break the dormancy of seeds and shorten breeding cycle, which can meet the demand for seedling supply of Polygonatum cyrtonema. In this study, transcriptome combined with metabolome was used to detect the changes of RNA level and metabolome level in the seed germination of Huangjing. We found that the DEGs were significantly enriched in the flavonoid synthesis and the phenylpropanoid metabolism pathway. DEGs was significantly up-regulated in the flavonoid (anthocyanin) synthesis pathway. The results of metabolites were also consistent with the RNA_sEq. Compared with the control, the accumulation of flavonoids significantly increased, while the hydroxycinnamyl significantly decreased in germinated seeds. Flavones are synthesized by the flavonoid pathway, which is part of phenylpropanoid metabolism[24]. Certain researches provided evidence to suggest that flavonoid play an important roles in plant defense against biotic and abiotic stresses, such as plant defense response to pathogen[25], oxidative damage[26] and UV stress[27] et al. However, rarely studies on the overall analysis of transcriptome and metabolome changes of flavonoid biosynthesis pathway in seed germination of Huangjing. Our results suggest that the DEGs and the metabolites of flavonoids synthesis pathway can promote seeds germination of Polygonatum cyrtonema.

Lignin Synthesis Associated With Seed Germination

The seed coat of Polygonatum cyrtonema seed is constituted by a layer of lignified cells[28], which prevents water absorption and has an inhibitory effect on seed germination. Lignin, plant secondary metabolism, which is a product of the phenylpropanoid pathway, has a physical barrier to seed germination. In present study, we found that DEGs and metabolisms were significantly down-regulated in the phenylpropanoid synthesis (lignin synthesis) pathway. Phenylpropanoid biosynthesis pathway 
followed by certain steps of the enzymes catalyzed. The key enzymes to regulate lignin synthesis, such as ammonia lyase (PAL), caffeoyl-CoA Omethyltransferase (CoMT), cinnamate 4-hydroxylase $(\mathrm{C} 4 \mathrm{H})$, 4coumarate-CoA ligase (4CL), cinnamoyl-CoA reductase (CCR), ferulic acid 5-hydroxylase ( $F 5 H)$ and cinnamyl alcohol dehydrogenase (CAD) were down-regulated in sprouted Huangjing seeds. Furthermore, compared with $\mathrm{CK}$, the metabolites were down-regulated significantly in this pathway (Fig. 8C). Finally, the products of three types of monolignols polymerize to form lignin $(G, S$, and $H$ ) will decreased in germinate Huangjing seeds. Therefore, the down-regulated DEGs and reduction of metabolites inhibits lignin synthesis in the phenylpropanoid synthesis pathway, which is also conducive to the Huangjing seeds to break through the seed coat and to break dormancy.

Plant Hormones Associated With Seed Germination

Germination is regulated by plant hormones[29]. Polygonatum cyrtonema seeds are affected by endogenous hormones and physiological post-ripening, which leads to low germination rate and difficult emergence of seeds. The study found that GA promotes seed germination and plays an important roles in regulating seed sprouting, plant growth and development[30-31]. In plant, GA is also related to induce the degradation of cell wall, which can promote endosperm degradation and to accelerate seed germination[32] and exogenous applications of GA was involved in breaking dormancy[33]. Abscisic acid (ABA) also plays an important role in regulating seed germination. However, compared with GA, ABA was proved to be an inhibitors that have reversed effect on seed germination[34]. ABA was involved in maintenance of seed dormancy, while GA and auxin were positive regulators for seed germination[35]. Similar to previous study, our results also found that GA- and auxin-related DEGs were significantly higher expressed in germinated seeds. However, the expression of DEGs associated with ABA synthesis was significantly down-regulated. Both GA and auxin can promote germination to break seed dormancy, while ABA promotes seed dormancy. Therefore, our results indicate that these DEGs play an important roles to regulate seed dormancy and germination.

\section{Starch And Sugar Metabolism Associated With Seed Germination}

In Polygonatum cyrtonema seeds, starch and proteins are stored in the endosperm to provide energy and substrates for germination. Seed germination is dependent on the decomposition of storage reserves in mature seeds, and the sugars from starch hydrolysis are the major source of energy for seeds sprout[36]. Huangjing seeds have a high dense of endosperm and a thick endosperm coat, which occupies by a large number of starch $(>73.7 \%$ )[37]. In present study, we suggest that Huangjing seeds germination were related to starch hydrolysis in endosperm. The decomposition of starch can provide energy during the seed germination[38]. a-amylase (EC 3.2.1.1) is the major enzyme that converts starch to oligosaccharides or glucose[39]. Thus, a-amylase activity play an important roles in plant seed germination[40]. During seed germination, the a-amylase gene expression and a-amylase activity (synthesis) were induced by GA synthesizing and transporting to the seed aleurone layer. Then, a-amylase is secreted into the endosperm to breaks down the stored starch into sugar[41]. Therefore, the activity of a-amylase is one of the indicators for judging seed germination, such as the seed germination of rice [42]. 
Our results demonstrate that a-amylase activity significantly increased in germination seed and the DEGs were significantly upregulated in the starch sugar metabolism pathway. This result indicates that hydrolysis of starch to monosaccharides can provides necessary energy for seed germinations.

\section{Conclusions}

In this study, we performed the metabolomics and transcriptomics analysis to reveal the underlying mechanisms of Polygonatum cyrtonema Hua to beak the seeds dormancy. A total of 56,628 unigenes were identified by RNA_seq and the average length of unigenes was $855 \mathrm{bp}$, the length of N50 was 1,127 bp. Compared with the CK, a total of 18,308 up-regulated DEGs and 9,709 down-regulated DEGs in the germinated seeds. A total of 637 metabolites were detected in Huangjing seeds by LC-MS. The results of RNA_seq and metabolomic indicate that phenylpropanoid and flavonoid biosynthesis pathway are important for seed germination. The metabolites flow of phenylalanine pathway was mainly to promote flavonoids synthesis and to inhibit the lignin synthesis during the seeds germination, which was beneficial to the germination of Polygonatum cyrtonema seeds. In addition, the results show that plant hormones (auxin, GA and $A B A$ ) and organic acids were involved in seed germination. In present study, we found that $A B A$ and organic acids were significantly inhibit seed germination. Moreover, the expression levels of unigenes were also consistent with the trends of a-amylase activity, indicating that the activity of a-amylase and sucrose metabolism might play an important roles in promoting seed germination. To study the association between transcriptomic and metabolic in seeds, we analyzed the connection of DEGs expression and metabolites. Certain key enzyme encoding DEGs were related to produce metabolites, this result reveal that these enzyme encoding DEGs and metabolites might be involved in seeds germination.

\section{Methods}

Plant materials

The Polygonatum cyrtonema Hua seeds (obtained from Chengdu Institute of Biology, Chinese Academy of Sciences, CIB, CAS) were used in this study, which undertook the formal identification by CIB, CAS. Mature Polygonatum cyrtonema Hua seeds were surface sterilized with $3 \% \mathrm{H}_{2} \mathrm{O}_{2}$ for 10 min, then vigorously rinsed with distilled water (>200 ml/per time) for 5 times. Remove the float seeds and air-dried, the plumply and shiny seeds were selected to cultivate in rotten leaf soil for experiments. Germination $(G)$ and non-germinating (CK) Polygonatum cyrtonema Hua seeds were collected after six months (each group with 3 biological replicates). All samples were frozen in liquid nitrogen and stored in a $-80^{\circ} \mathrm{C}$ refrigerator for metabolome analyze and transcriptome sequencing.

Metabolome analyze of Polygonatum cyrtonema Hua seeds

The freeze-dried seed was crushed using a mixer mill (MM 400, Retsch) with a zirconia bead for 1.5 min at $30 \mathrm{~Hz} .100 \mathrm{mg}$ powder was weighted and extracted overnight at $4{ }^{\circ} \mathrm{C}$ with $1.0 \mathrm{ml} 70 \%$ aqueous 
methanol. Following centrifugation at 10, $000 \mathrm{~g}$ for $10 \mathrm{~min}$, the extracts were absorbed (CNWBOND Carbon-GCB SPE Cartridge, 250 mg, 3 ml; ANPEL, Shanghai, China) and filtrated (SCAA-104, $0.22 \mu \mathrm{m}$ pore size; ANPEL, Shanghai, China) before LC-MS analysis.

HPLC Conditions

The extracts sample were analyzed using an LC-ESI-MS/MS system (HPLC, Shim-pack UFLC SHIMADZU CBM30A system; MS, Applied Biosystems 6500 Q TRAP). The analytical conditions were as follows, HPLC: column, Waters ACQUITY UPLC HSS T3 C18 $(1.8 \mu \mathrm{m}, 2.1 \mathrm{~mm} * 100 \mathrm{~mm})$; solvent system, water (0.04\% acetic acid): acetonitrile (0.04\% acetic acid); gradient program,95:5V/V at $0 \mathrm{~min}, 5: 95 \mathrm{~V} / \mathrm{V}$ at $11.0 \mathrm{~min}, 5: 95 \mathrm{~V} / \mathrm{V}$ at $12.0 \mathrm{~min}, 95: 5 \mathrm{~V} / \mathrm{V}$ at $12.1 \mathrm{~min}, 95: 5 \mathrm{~V} / \mathrm{V}$ at $15.0 \mathrm{~min}$; flow rate, $0.40 \mathrm{ml} / \mathrm{min}$; temperature, $40^{\circ} \mathrm{C}$; injection volume: $2 \mu \mathrm{l}$. The effluent was alternatively connected to an ESI-triple quadrupole-linear ion trap (Q TRAP)-MS.

\section{ESI-Q TRAP-MS/MS}

LIT and triple quadrupole (QQQ) scans were acquired on a triple quadrupole-linear ion trap mass spectrometer (Q TRAP), API 6500 Q TRAP LC/MS/MS System, equipped with an ESI Turbo lon-Spray interface, operating in a positive ion mode and controlled by Analyst 1.6 software (AB Sciex). The ESI source operation parameters were as follows: ion source, turbo spray; source temperature $500{ }^{\circ} \mathrm{C}$; ion spray voltage (IS) $5500 \mathrm{~V}$; ion source gas I (GSI), gas II(GSII), curtain gas (CUR) were set at 55, 60, and $25.0 \mathrm{psi}$, respectively; the collision gas(CAD) was high. Instrument tuning and mass calibration were performed with 10 and $100 \mu \mathrm{mol} / \mathrm{L}$ polypropylene glycol solutions in QQQ and LIT modes, respectively. QQQ scans were acquired as MRM experiments with collision gas (nitrogen) set to $5 \mathrm{psi}$. DP and CE for individual MRM transitions was done with further DP and CE optimization. A specific set of MRM transitions were monitored for each period according to the metabolites eluted within this period. RNA isolation and cDNA library construction for RNA Sequencing

Total RNA of Polygonatum cyrtonema Hua seeds were extracted using the mirVana miRNA Isolation Kit (Ambion) following the manufacturer's protocol. RNA integrity was evaluated using the Agilent 2100 Bioanalyzer (Agilent Technologies, Santa Clara, CA, USA). The samples with RNA Integrity Number (RIN) $\geq 7$ were subjected to the subsequent analysis. Total RNA from the seeds were to break into short fragments at a suitable temperature in the thermomixer. Then purified fragment mRNA was used to synthesize first strand cDNA and second strand cDNA. The libraries were constructed using TruSeq Stranded mRNA LTSample Prep Kit (Illumina, San Diego, CA, USA) according to the manufacturer's instructions. Then 6 cDNA libraries were sequenced on the lllumina sequencing platform (HiSeqTM 2500 or Illumina HiSeq X Ten) and 125 bp/150 bp paired-end reads were generated. Raw data (raw reads) were processed using Trimmomatic[43]. The reads containing ploy- $\mathrm{N}$ and the low quality reads were removed too btain the clean reads. After removing adaptor and low quality sequences, the clean reads were assembled into expressed sequence tag clusters (contigs) and de novo assembled into transcript by using Trinity[44] in paired-end method. The longest transcript was choose as a unigene based the similarity and length of a sequence for subsequent analysis. 
Functional annotation of differential expression unigenes (DEGs)

The function of the unigenes was annotated by alignment of the unigenes with the NCBI non-redundant (NR), SwissProt, and Clusters of orthologous groups for eukaryotic complete genomes (KOG) data bases using Blastx[45] with a threshold E-value of $10^{-5}$. The proteins with the highest hits to the unigenes were used to assign functional annotations thereto. Base on the SwissProt annotation,Gene ontology (GO) classification was performed by the mapping relation between SwissProt and GO term. The unigenes were mapped to the Kyoto Encyclopedia of Genes and Genomes (KEGG)[46] database to annotate their potential metabolic pathways.

FPKM[47] and read counts value of each unigene was calculated using bowtie2[48] and eXpress[49]. DEGs were identified using the DESeq[50] functions estimate SizeFactors and nbinom Test. P value $<0.05$ and fold Change $>2$ or fold Change $<0.5$ was set as the threshold for significantly differential expression. Hierarchical cluster analysis of DEGs was performed to explore transcripts expression pattern. GO enrichment and KEGG pathway enrichment analysis of DEGs were respectively performed using $\mathrm{R}$ based on the hypergeometric distribution.

\section{Determination Of Alpha-amylase Activity}

Germination and CK Polygonatum cyrtonema Hua seeds or radicle were collected to determine a-amylase activity. Add $1 \mathrm{~mL}$ distilled water in $0.1 \mathrm{~g}$ plant sample to homogenize and pour the homogenate into a centrifuge tube at room temperature for extraction for $20 \mathrm{~min}$ and shaking to fully extract; centrifuge (8000 rpm) at $25^{\circ} \mathrm{C}$ for $10 \mathrm{~min}$, then suck the supernatant, add distilled water to $10 \mathrm{~mL}$, and shaking to obtain the amylase stock solution. The a-amylase activity was determined by alpha-amylase assay kit (Suzhou Geruisi Biological Technology Co., Ltd., China), follow the manufacturer's protocol to detected and calculated a-amylase activity.

qRT-PCR analysis

Eighteen DEGs were selected for qRT-PCR analysis, and GAPDH was used as the internal reference gene. The primers were designed based on the sequence of genes using Primer 5.0 and are listed in Table S1. The RNA extracted from Polygonatum cyrtonema Hua were used to synthesize first-strand cDNA with HiScriptll Q RT SuperMix (Vazyme, R223-1) following the manufacturer's instructions. The qRT-PCR was performing with SYBR ${ }^{8}$ Green PCR kit (Qiagen, 204054) according to the manufacturer's instructions. The experimental conditions were set as follows: 45 cycles at $95^{\circ} \mathrm{C}$ for $20 \mathrm{~s}, 55^{\circ} \mathrm{C}$ for $20 \mathrm{~s}$, and $72{ }^{\circ} \mathrm{C}$ for $20 \mathrm{~s}$. All genes were repeated 3 times in this study. The mRNA expression level of the genes were calculated with the $2^{-\Delta \Delta C t}$ method[51]. Each plant sample was repeated 3 times. The correlation between RNA-Seq and qRT-PCR results was analyzed using these values $\mathrm{R}$ package version 3.1 .3 (http://cran.rproject.org/). The normalized values of relative expression and RPKM values were calculated using $\log _{2}$ (fold change) measurements. 
Data of three biological repeats were analyzed using GraphPad Prism 5, Excel 2013 and SPSS 20.0 and rendered as means \pm SD. One-way ANOVA followed by Tukey's significant difference test at $p<0.05$. All data had 3 biological repeats. Differentially expressed genes were defined as genes with FDR $<0.001$ and fold change $>2$-fold. A p-value $<0.05$ was considered significant when identifying enriched GO terms, and a $p$-value $<0.05$ was considered indicative of significantly enriched KEGG pathways.

\section{Abbreviations}

Germination (G), gibberellin acid (GA), abscisic acid (ABA), liquid chromatography-MS (LC/MS), next generation sequencing (NGS), differential expression unigenes (DEGs), NCBI non-redundant (NR), eukaryotic complete genomes (KOG), gene ontology (GO), Kyoto Encyclopedia of Genes and Genomes (KEGG), ammonia lyase (PAL), cinnamate 4-hydroxylase (C4H), 4-coumarate-CoA ligase (4CL), cinnamoyl-CoA reductase (CCR), caffeoyl-CoA Omethyltransferase (CoMT), ferulic acid 5-hydroxylase $(\mathrm{F} 5 \mathrm{H})$, cinnamyl alcohol dehydrogenase (CAD).

\section{Declarations}

Ethics approval and consent to participate

Not applicable.

\section{Consent for publication}

Not applicable.

\section{Availability of data and materials}

The dataset and materials presented in the investigation is available by request from the corresponding author.

\section{Competing interests}

The authors declare that they have no competing interests. There was no competing Interests in this work.

\section{Funding}


This work was supported by the Sichuan Science and Technology Plan Project (Grant numbers 2017NFP0053). National Survey of Traditional Chinese Medicine Resources (Grant numbers GZY-KJS2018-004). Innovation Academy for Seed Design, CAS. The funders had no role in the study design, data collection, and analysis, decision to publish or preparation of the manuscript.

\section{Authors' Contributions}

$\mathrm{YW}$ and $\mathrm{LZ}$ designed the study and provided plant material. $\mathrm{RL}$ and $\mathrm{JL}$ performed the experiments and data analyses. $R L$ and $J L$ contributed to the development of material and writing of the manuscript. $R L$, $\mathrm{JL}, \mathrm{JYX}$ and $\mathrm{CHZ}$ contributed by collecting the samples. JYX, CHZ, MZ, MD, MXW and YFL were read this article and modified. All authors read and approved the final manuscript.

\section{Acknowledgements}

We thanks Qiaona Zhang (Renqiu Affiliated School of Beijing Normal University), Jun Kuang, Ximin Zou, Xinglin Huang and Junwen Yang for technical support.

\section{Authors' Information}

${ }^{1}$ Chengdu Institute of Biology, Chinese Academy of Sciences, Chengdu 610041, China

2 University of Chinese Academy of Sciences, Beijing 100049, China

*Correspondence: 13555809468@163.com; wuyu@cib.ac.cn

${ }^{\dagger}$ These authors contributed equally to this work.

\section{References}

1. Ma, Y.L.; Zhang, Y.S.; Zhang, F.; Zhang, Y.Y.; Thakur, K.; Zhang, J.G.; Wei, Z.J. Methyl protodioscin from Polygonatum sibiricum inhibits cervical cancer through cell cycle arrest and apoptosis induction. Food Chem. Toxicol. 2019, 132, 110655.

2. Yuan, M.A.; Kong, X.J.; Chen, Y.H.; Liu, X.H.; Cao, C.X. Study on interplanting cultivation techniques of sweet corn with different planting densities and sealwort. Horticul. \& Seed. 2012, 12, 3-5.

3. Zhao, Z.; Pang, Y.X.; Yuan, Y.; Fu, M.X.; Liu, S.Q.; Cao, D.T.; Xian, F.R. Study on the seed reproduces technology of medicinal crop rhizome polygonati. Seed. 2005, 24, 11-13. 
4. Huang, P.L.; Gan, K.H.; Wu, R.R.; Lin, C.N. Benzoquinones, a homoisoflavaone and other constituents from Polygonatum altelobatum. Phytochemistry. 1997, 44, 1369-1373.

5. Wu, S.R.; Li, Y.Y.; Xiao, S『Experimental study of the effect of polygona polysaccharose on hypedipoidemia $₫$ Chin. J. New Drugs. 2003, 12, 108-110区

6. Lisec, J.; Schauer, N.; Kopka, J.; Willmitzer, L.; Fernie, A.R. Gas chromatography mass spectrometrybased metabolite profiling in plants. Nat. Protoc. 2006, 1, 387-396.

7. Ponnaiah, M.; Gilard, F.; Gakière, B.; El-Maarouf-Bouteau, H.; Bailly, C. Regulatory actors and alternative routes for Arabidopsis seed germination are revealed using a pathway-based analysis of transcriptomic datasets. Plant J. 2019, 99, 163-175.

8. Fait, A.; Angelovici, R.; Less, H.; Ohad, I.; Urbanczyk-Wochniak, E.; Fernie, A.R.; Galili, G. Arabidopsis seed development and germination is associated with temporally distinct metabolic switches. Plant Physiol. 2006, 142, 839-854.

9. Sreenivasulu, N.; Usadel, B.; Winter, A.; Radchuk, V.; Scholz, U.; Stein, N.; Weschke, W.; Strickert, M.; Close, T.J.; Stitt, M.; Graner, A.; Wobus, U. Barley grain maturation and germination: metabolic pathway and regulatory network commonalities and differences highlighted by new MapMan/PageMan profiling tools. Plant Physiol. 2008, 146, 1738-1758.

10. Hu, C.; Tohge, T.; Chan, S.A.; Song, Y.; Rao, J.; Cui, B.; Lin, H.; Wang, L.; Fernie, A.R.; Zhang, D.; et al. Identification of conserved and diverse metabolic shifts during rice grain development. Sci. Rep. 2016, 10, 20942.

11. Mohammad, M.; D.L., Smithc. Plant hormones and seed germination. Environ. Exp. Bot. 2014, 99, 110-121.

12. Penfield, S.; Li, Y.; Gilday, A.D.; Graham, S.; Graham, I.A. Arabidopsis ABA INSENSITIVE4 regulates lipid mobilization in the embryo and reveals repression of seed germination by the endosperm. Plant Cell. 2006, 18, 1887-1899.

13. Holdsworth, M.J.; Bentsink, L.; Soppe, W.J. Molecular networks regulating Arabidopsis seed maturation, after-ripening, dormancy and germination. New Phytol. 2008, 179, 33-54.

14. Sheng, Y.; Xiao, H.; Guo, C.; Wu, H.; Wang, X. Effects of exogenous gamma-aminobutyric acid on aamylase activity in the aleurone of barley seeds. Plant Physiol. Biochem. 2018, 127, 39-46.

15. Liu, L.; Xia, W.; Li, H.; Zeng, H.; Wei, B.; Han, S.; Yin, C. Salinity Inhibits Rice Seed Germination by Reducing a-Amylase Activity via Decreased Bioactive Gibberellin Content. Front. Plant Sci. 2018, 9, 275.

16. Kaneko, M.; Itoh, H.; Ueguchi-Tanaka, M.; Ashikari, M.; Matsuoka, M. The alpha-amylase induction in endosperm during rice seed germination is caused by gibberellin synthesized in epithelium. Plant Physiol. 2002, 128, 1264-70.

17. Ma, Z.; Marsolais, F.; Bernards, M.A.; Sumarah, M.W.; Bykova, N.V.; Igamberdiev, A.U. Glyoxylate cycle and metabolism of organic acids in the scutellum of barley seeds during germination. Plant Sci. 2016, 248, 37-44. 
18. Aisyah, S.; Vincken, J.P.; Andini, S.; Mardiah, Z.; Gruppen, H5. Compositional changes in (iso)flavonoids and estrogenic activity of three edible Lupinus species by germination and Rhizopuselicitation. Phytochemistry. 2016, 122, 65-75.

19. Zhou, X.L.; Chen, Z.D.; Zhou, Y.M.; Shi, R.H.; Li, Z.J. The Effect of Tartary Buckwheat Flavonoids in Inhibiting the Proliferation of MGC80-3 Cells during Seed Germination. Molecules. 2019, 24(17).

20. Bryant, F.M.; Hughes, D.; Hassani-Pak, K.; Eastmond, P.J. Basic LEUCINE ZIPPER TRANSCRIPTION FACTOR67 Transactivates DELAY OF GERMINATION1 to Establish Primary Seed Dormancy in Arabidopsis. Plant Cell. 2019, 31, 1276-1288.

21. Ponnaiah, M.; Gilard, F.; Gakière, B.; El-Maarouf-Bouteau, H.; Bailly, C. Regulatory actors and alternative routes for Arabidopsis seed germination are revealed using a pathway-based analysis of transcriptomic datasets. Plant J. 2019, 99, 163-175.

22. Han, C.; Zhen, S.; Zhu, G.; Bian, Y.; Yan, Y. Comparative metabolome analysis of wheat embryo and endosperm reveals the dynamic changes of metabolites during seed germination. Plant Physiol. Biochem. 2017, 115, 320-327.

23. Baskin, C.C.; Baskin, J.M. A classification system for seed dormancy. Seed Sci. Research. 2004, 14, 116.

24. Falcone, Ferreyra, M.L.; Rius, S.P.; Casati, P. Flavonoids: Biosynthesis, biological functions, and biotechnological applications. Front. Plant Sci. 2012, 28, 3:222.

25. La, Camera, S.; Gouzerh, G.; Dhondt, S.; Hoffmann, L.; Fritig, B.; Legrand, M.; Heitz, T. Metabolic reprogramming in plant innate immunity: the contributions of phenylpropanoid and oxylipin pathways. Immunol. Rev. 2004, 198, 267-284.

26. Yin, R.; Ulm, R. How plants cope with UV-B: from perception to response. Curr. Opin. Plant Biol. 2017, $37,42-48$.

27. Wang, F.; Xu, Z; Fan, X.; Zhou, Q.; Cao, J.; Ji, G. Transcriptome Analysis Reveals Complex Molecular Mechanisms Underlying UV Tolerance of Wheat (Triticum aestivum, L.). J. Agric. Food Chem. 2019, $67,563-577$.

28. Wang, J.L.; Chang, H.; Zhou, Z.L.; Chen, Y.Y.; Sun, Y.Y.; Gou, H.B.; Zhang, Y. J. Development anatomy of Polygonatum sibiricum Red. During the seed germination process. Acta. Bot. Boreal-Occident Sin. 2013, 33, 1584-1588.

29. Weitbrecht, K.; Müller, K.; Leubner-Metzger, G. First off the mark: early seed germination. J. Exp. Bot. 2011, 62, 3289-3309.

30. Meng, Y.; Chen, F.; Shuai, H.; Luo, X.; Ding, J.; Tang, S.; Xu, S.; Liu, J.; Liu, W.; Du, J.; et al. Karrikins delay soybean seed germination by mediating abscisic acid and gibberellin biogenesis under shaded conditions. Sci. Rep. 2016, 23, 22073.

31. Richards, D.E.; King, K.E.; Ait-Ali, T.; Harberd, N.P. How gibberellin regulates plant growth and development: a molecular genetic analysis of gibberellin signaling. Annu. Rev. Plant Physiol. Plant Mol. Biol. 2001, 52, 67-88. 
32. Leubner-Metzger, G®Functions and regulation of $\beta-1,3$ glucanase during seed germination $\llbracket$ dormancy release and after ripening. Seed Sci. Research. 2003, 13, 17-34区

33. Frigerio, M.; Alabadí, D.; Pérez-Gómez, J.; García-Cárcel, L.; Phillips, A.L.; Hedden, P.; Blázquez, M.A. Transcriptional regulation of gibberellin metabolism genes by auxin signaling in Arabidopsis. Plant Physiol. 2006, 142, 553-563.

34. Shu, K.; Liu, X.D.; Xie, Q.; He, Z.H. Two faces of one seed: hormonal regulation of dormancy and germination. Mol. Plant. 2016, 9, 34-45.

35. Finkelstein, R.R.; Gampala, S.S.; Rock, C.D. Abscisic acid signaling in seeds and seedlings. Plant Cell. 2002, 14, S15-45.

36. Beck, E.; Ziegler, P. Biosynthesis and degradation of starch in higher plants. Annu. Rev. Plant Physiol. Plant Mol. Biol. 1989, 40, 95-117.

37. Sun, W.S.; Chen, W.X.; Zhu, L.X.; Qin, N.; Liu, Y.Y.; Qu, H.Y.; Zhen, M.; Sun, M.T.; Ge, C.M. Effects of lowtemperature and gibberellin on seed germination characteristics of Polygonatum sibiricum. ShangDong Agric. Sci. 2019, 51, 64-67.

38. Chen, F.; Bradford, K.J. Expression of an expansion is associated with endosperm weakening during tomato seed germination. Plant Physiol. 2000, 124, 1265-1274.

39. Sun, Z.; Henson, C.A. A quantitative assessment of the importance of barley seed a-amylase, bamylase, debranching enzyme, and a-glucosidase in starch degradation. Arch. Biochem. Biophys. 1991, 284, 298-305.

40. Karrer, E.E.; Chandler, J.M.; Foolad, M.R.; Rodriguez, R.L. Correlation between a-amylase gene expression and seedling vigor in rice. Euphytica. 1992, 66, 163-169.

41. Kaneko, M.; Itoh, H.; Ueguchi-Tanaka, M.; Ashikari, M.; Matsuoka, M. The a-amylase induction in endosperm during rice seed germination is caused by gibberellin synthesized in epithelium. Plant Physiol. 2002, 128, 1264-1270.

42. Liu, L.; Xia, W.; Li, H.; Zeng, H.; Wei, B.; Han, S.; Yin, C. Salinity inhibits rice seed germination by reducing a-amylase activity via decreased bioactive gibberellin content. Front. Plant Sci. 2018, 9, 275284.

43. Bolger, A.M.; Lohse, M.; Usadel, B. Trimmomatic: aflexible trimmer for Illumina sequence data. Bioinformatics. 2014, 30, 2114-2120.

44. Grabherr, M.G.; Haas, B.J.; Yassour, M.; Levin, J.Z.; Thompson, D.A.; Amit, I.; Adiconis, X.; Fan, L.; Raychowdhury, R.; Zeng, Q.; et al. Trinity: reconstructing a full-length transcriptome without a genome from RNA-Seq data. Nat. Biotechnol. 2011, 29, 644-652.

45. Altschul, S.F.; Gish, W.; Miller, W.; Myers, E.W.; Lipman, D.J. Basic local alignment search tool. J. Mol. Biol. 1990, 215, 403-410.

46. Kanehisa, M.; Araki, M.; Goto, S.; Hattori, M.; Hirakawa, M.; Itoh, M.; Katayama, T.; Kawashima, S.; Okuda, S.; Tokimatsu, T.; et al. KEGG for linking genomes to life and the environment. Nucleic. Acids. Res. 2008, 36, D480-484. 
47. Trapnell, C.; Williams, B.A.; Pertea, G.; Mortazavi, A.; Kwan, G.; van, Baren, M.J.; Salzberg, S.L.; Wold, B.J.; Pachter, L. Transcript assembly and quantification by RNA-Seq reveals unannotated transcripts and isoform switching during cell differentiation. Nat. Biotechnol. 2010, 28, 511-515.

48. Langmead, B.; Salzberg, S.L. Fast gapped-read alignment with Bowtie2. Nat. Methods. 2012, 9, 357359.

49. Roberts, A.; Pachter, L. Streaming fragment assignment for real-time analysis of sequencing experiments. Nat. Methods. 2013, 10, 71-73.

50. Anders, S. and W. Huber, Differential expression of RNA-Seq data at the gene level-the DESeq package. EMBL, 2013.

51. chmittgen, T.D.; Livak, K.J. Analyzing real-time PCR data by the comparative C-T method. Nat. Protoc. 2008, 3, 1101-1108.

\section{Additional Files}

Table S1. List of primers used for the relative quantification of gene transcripts (XLSX $11 \mathrm{~kb}$ ).

Supplementary File1. The data of DEGs in the germinated and CK seeds (XLSX $4351 \mathrm{~kb}$ ). Supplementary File2. The top ten of KEGG enrichment of DEGs in Polygonatum cyrtonema seeds. (XLSX $10 \mathrm{~kb}$ ). Supplementary File3. The data of DEGs enrichment in flavonoid biosynthesis (Ko00941) (XLSX $4941 \mathrm{~kb}$ ). Supplementary File4. The data of DEGs in starch and sucrose metabolism pathway (XLSX $13 \mathrm{~kb}$ ). Supplementary File5. The data of DEGs expression enriched in auxin, GA and ABA biosynthesis pathway (XLSX $18 \mathrm{~kb}$ ). Supplementary File6. The data of differentially metabolites in Polygonatum cyrtonema (XLSX $83 \mathrm{~kb}$ ). Supplementary File7. The data of differentially metabolites of flavonoids, hydroxycinnamyl and organic acid in Polygonatum cyrtonema (XLSX $14 \mathrm{~kb}$ ).

\section{Figures}



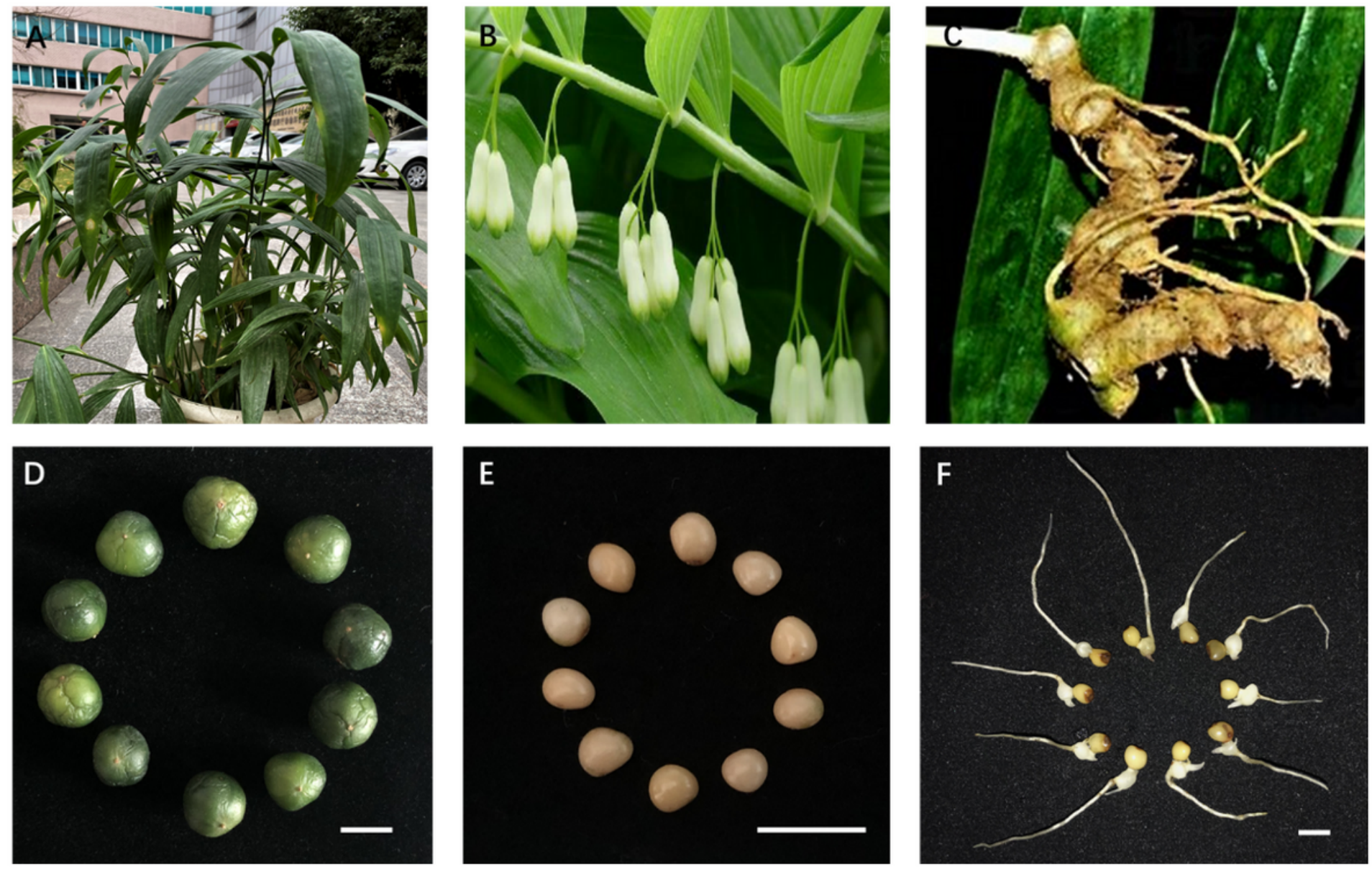

Figure 1

The plant morphology $(A)$, flower $(B)$, tubers $(C)$, fruits $(D)$, seeds $(E)$ and germinated seeds $(F)$ of Polygonatum cyrtonema Hua
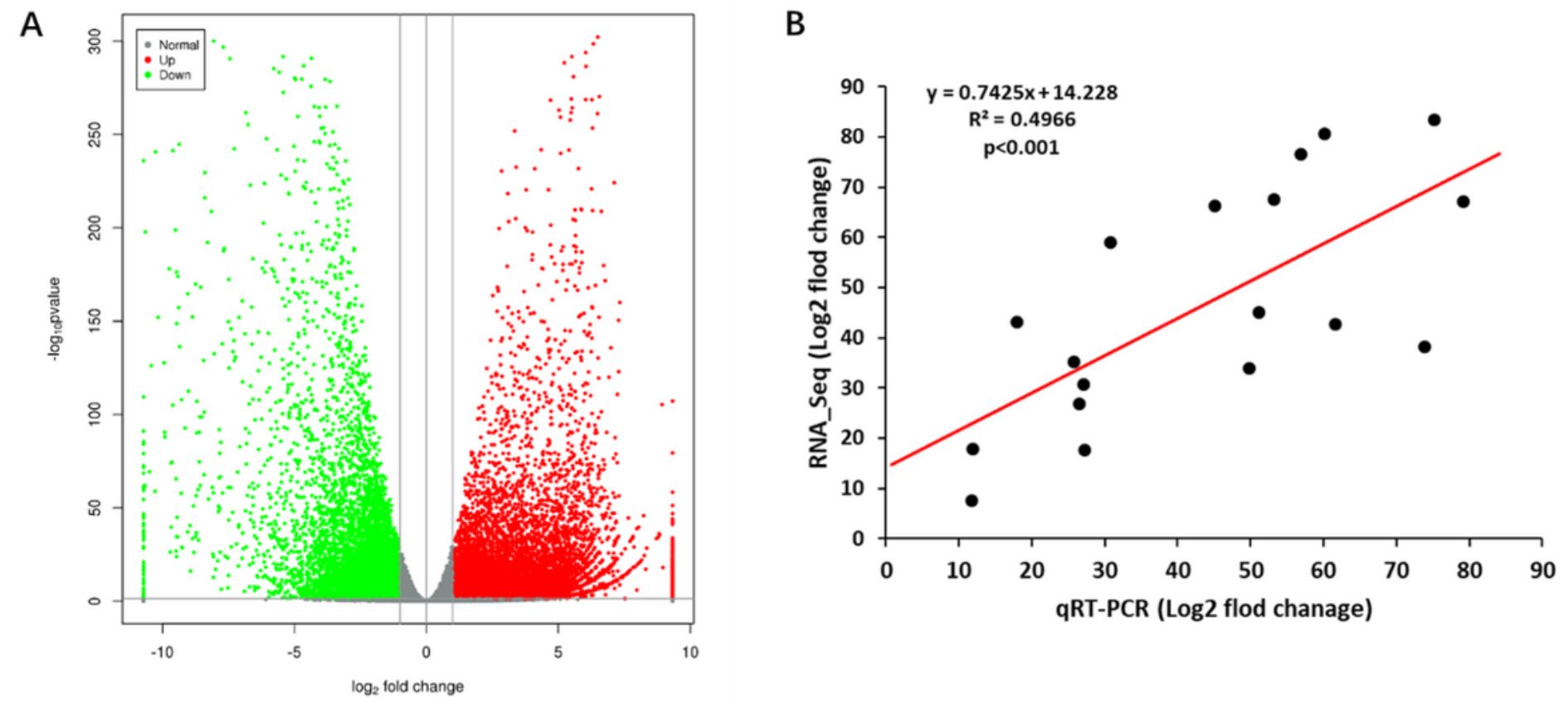
Figure 2

Volcano plots of differentially expressed unigenes (DEGs) between germinated and control seeds in Polygonatum cyrtonema Hua (A); and correlation between qRT-PCR and RNA_seq for the 18 genes (B). Each point represents a fold change value of gene expression level in Polygonatum cyrtonema Hua.

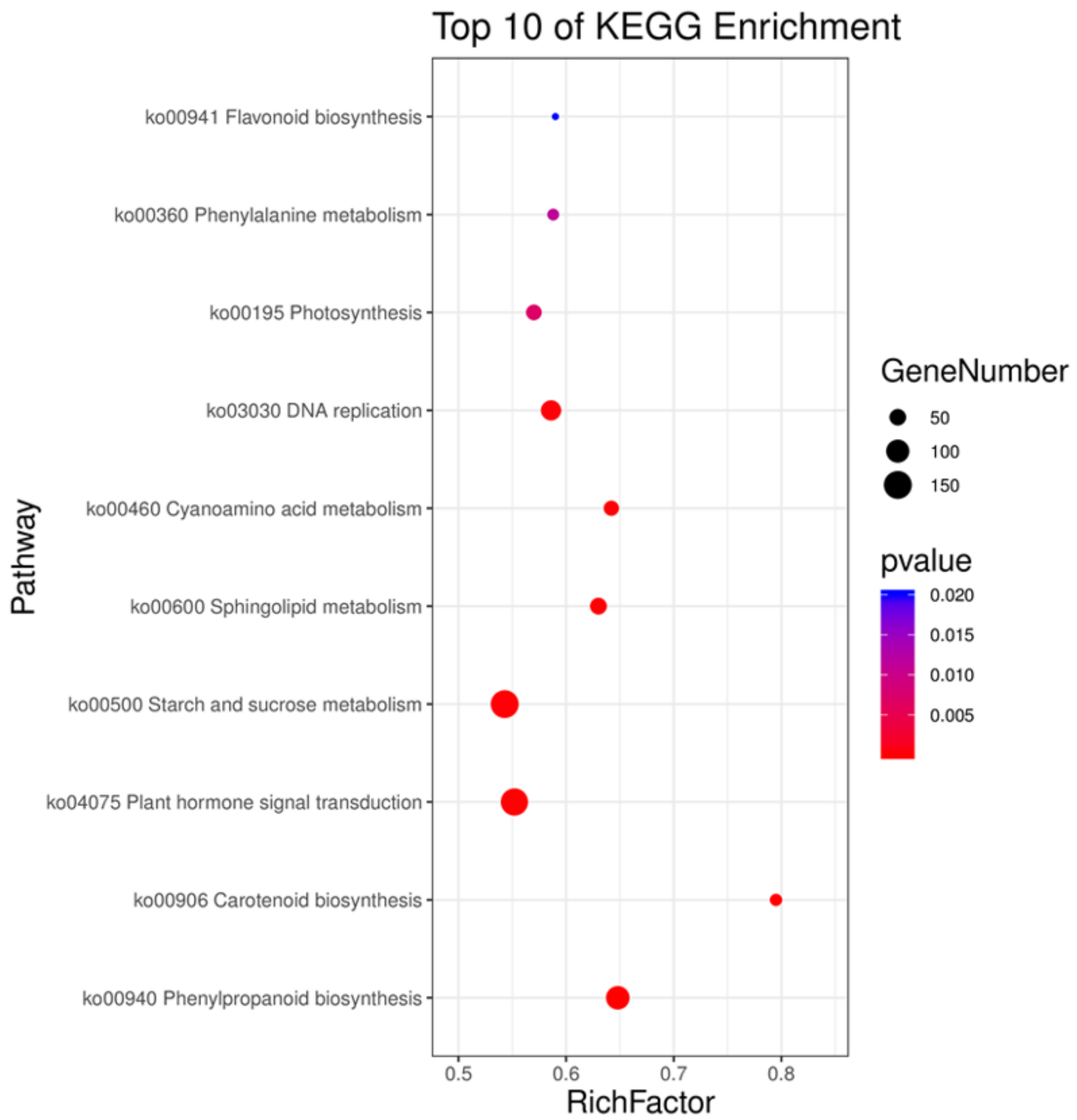

\section{Figure 3}

The top ten of KEGG enrichment of differentially expressed unigenes (DEGs) in Polygonatum cyrtonema Hua seeds. 
A

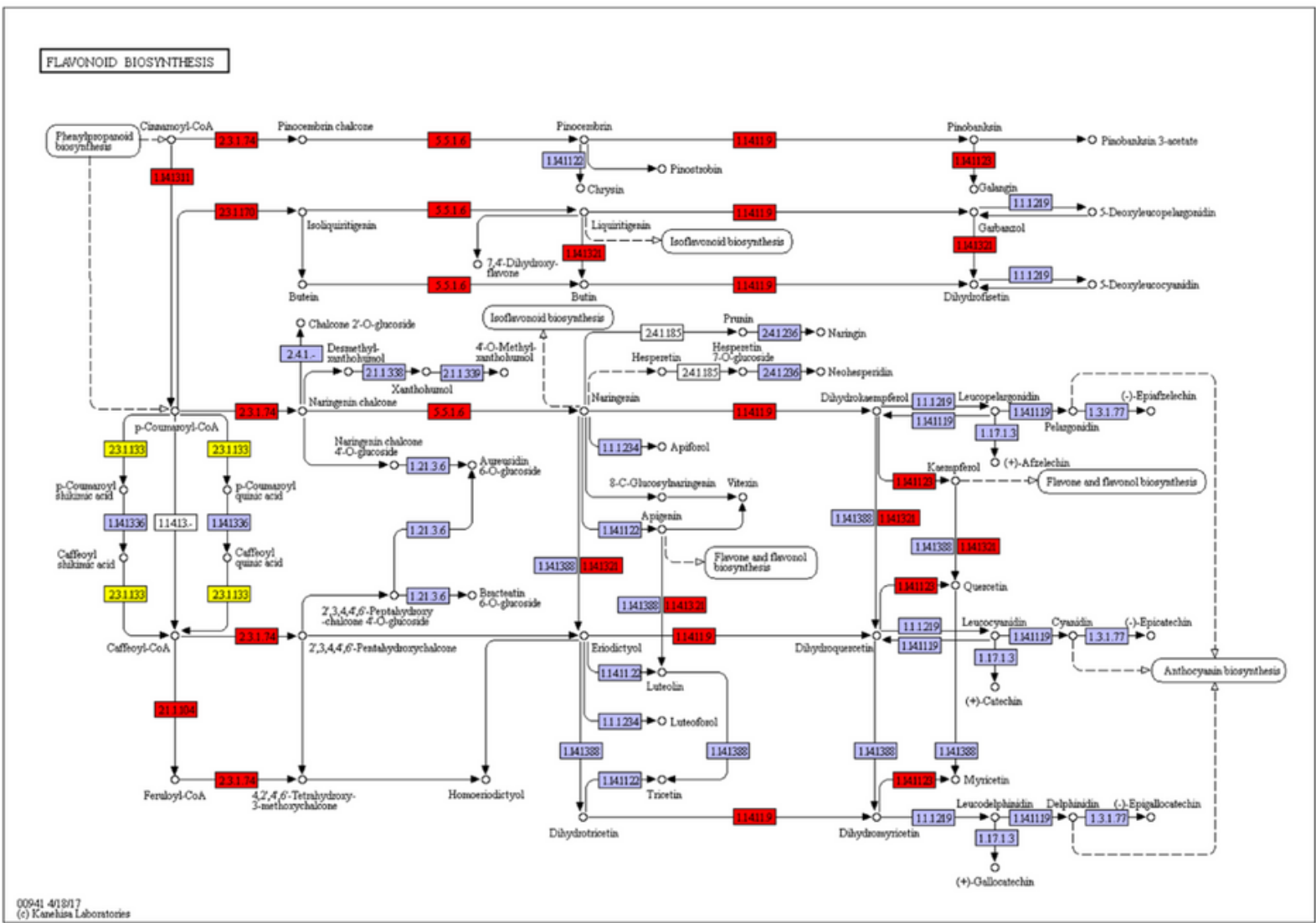

B

CHS-1

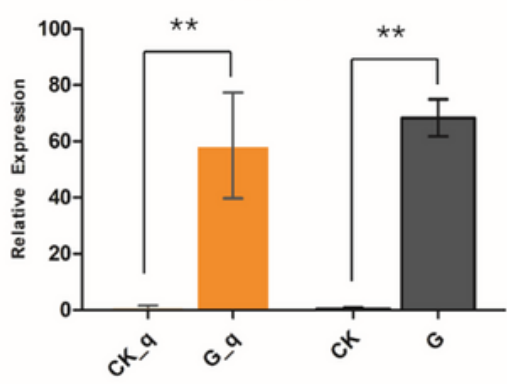

CHS-2
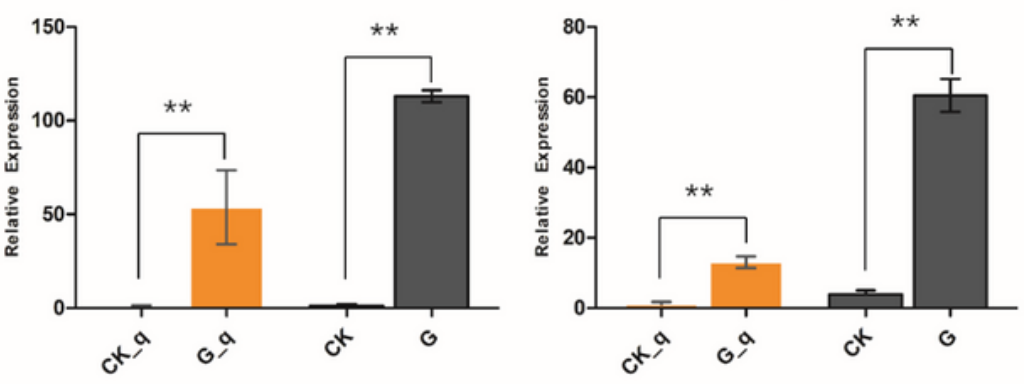

$\mathrm{F} 3 \mathrm{H}-1$
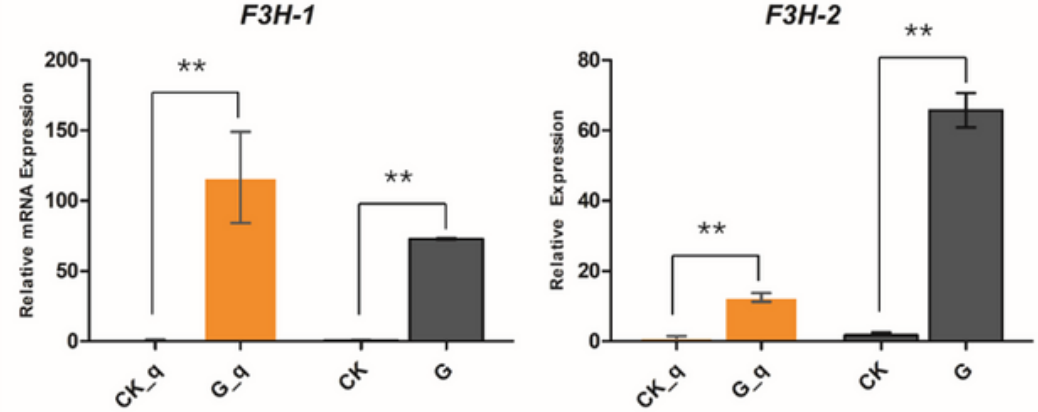

FLS

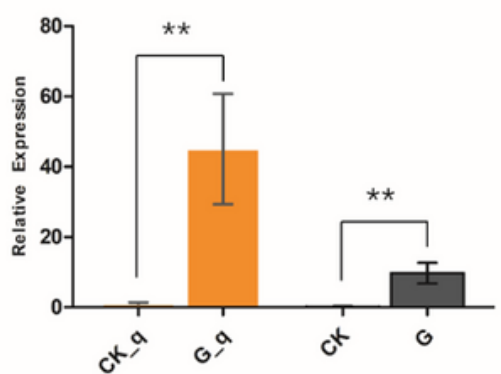

\section{Figure 4}

The expression of DEGs enrichment in flavonoid biosynthesis (Ko00941) in G vs CK seeds (A), genes marked with red indicate up-regulated, yellow has both up- and down-regulated genes in germinated seeds. The expression of flavonoid biosynthesis pathway genes by qPCR determination(B), expression levels estimated using log2(fold change) for each transcript $(* \star p<0.01)$. In the histogram, CK_q and G_q represent qPCR analysis; CK and G represent the FPKM value of RNA_seq. 
A
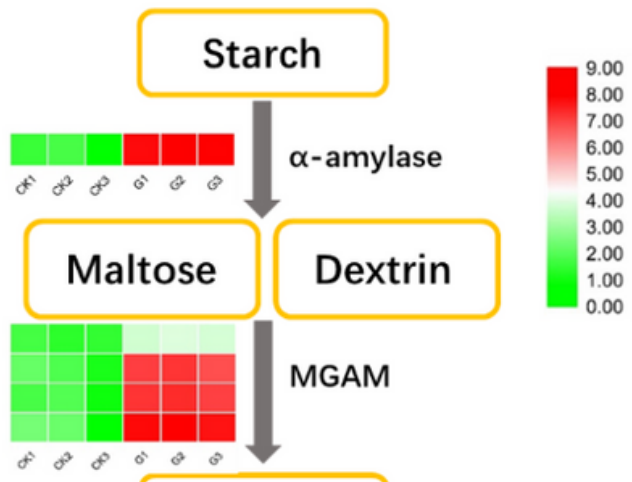

\section{D-Glucose}

$\mathrm{HK}$

\section{D-Glucose-6p}

C

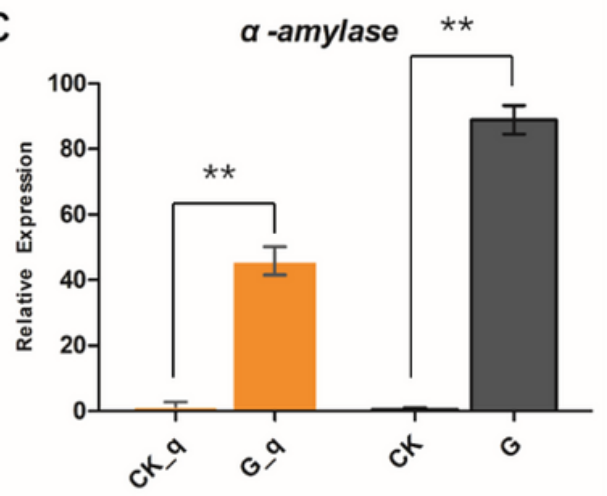

E3.2.1.20.

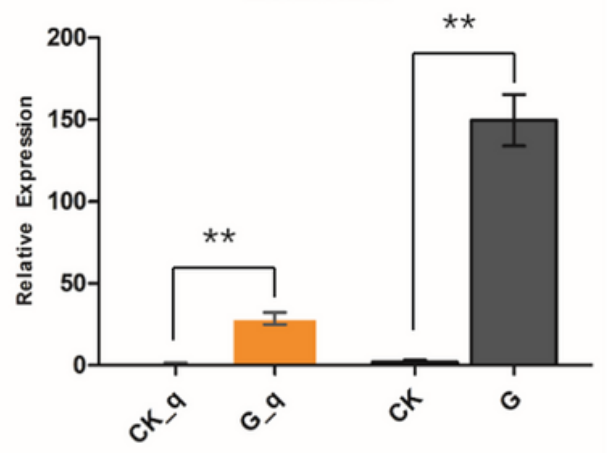

B

$\alpha$-amylase activity

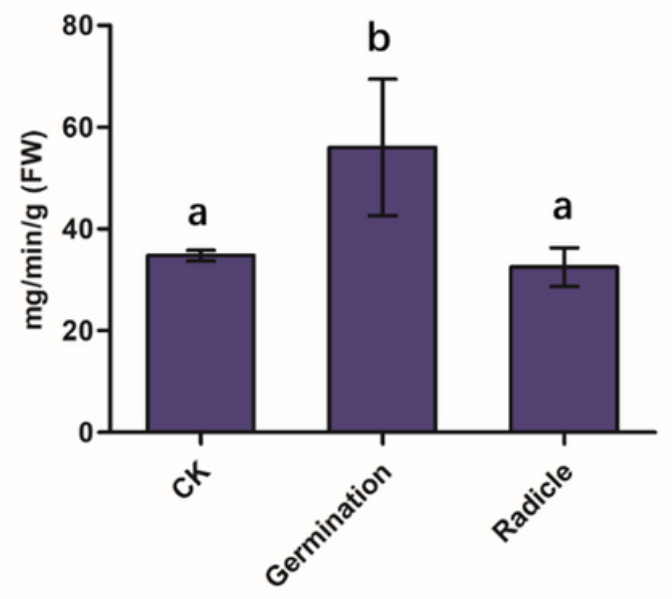

E3.2.1.20
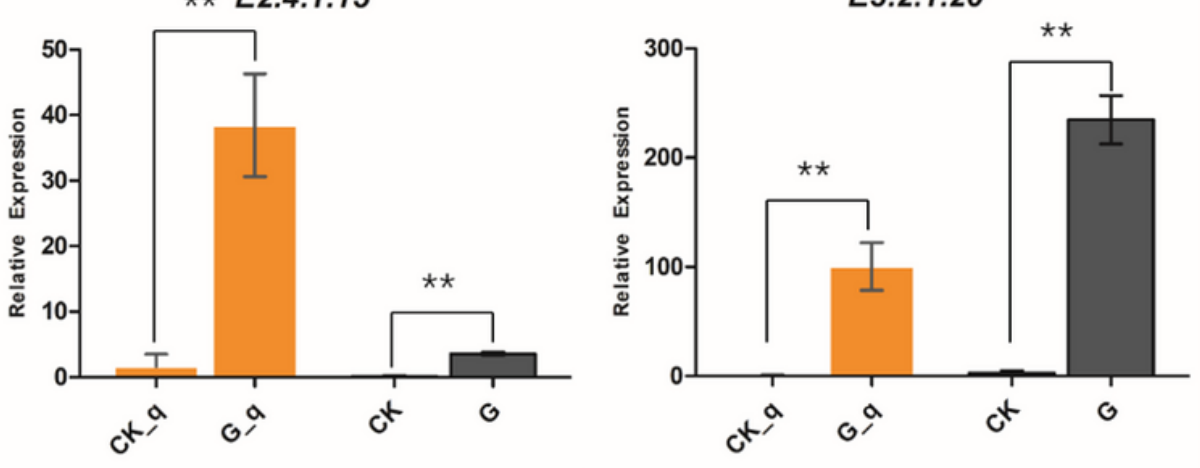

E3.2.1.21

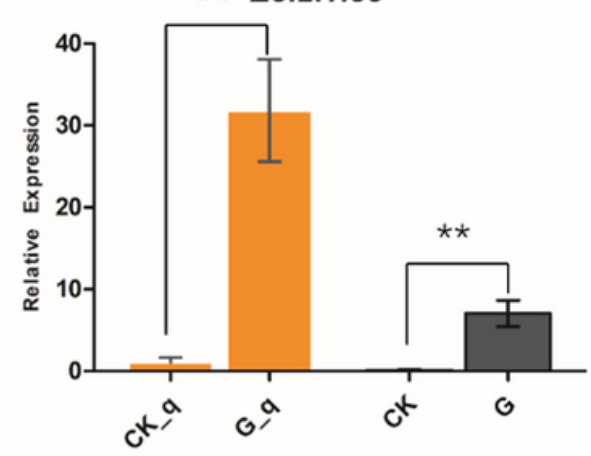

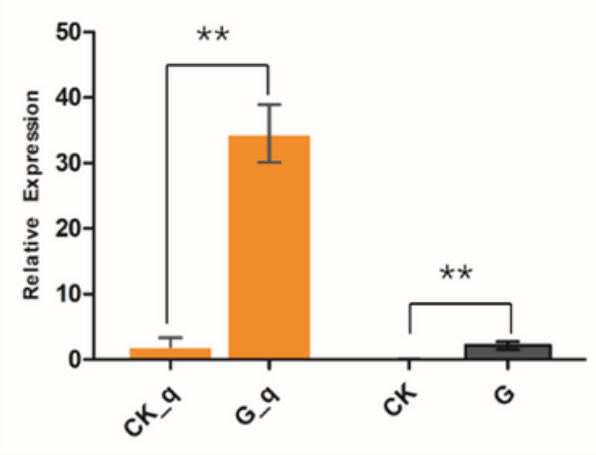

Figure 5

The expression of starch and sucrose metabolism pathway genes in Polygonatum cyrtonema Hua (A), expression levels are indicated by the heatmap at $\mathrm{G}$ and CK group, estimated using log2(fold change) for each transcript. The a-amylase activity (B) and the enzyme genes determined by qRT-PCR (C). In the histogram, CK_q and G_q represent qPCR analysis; CK and G represent the FPKM value of RNA_seq $\left({ }^{\star \star} p<0.01\right)$. 


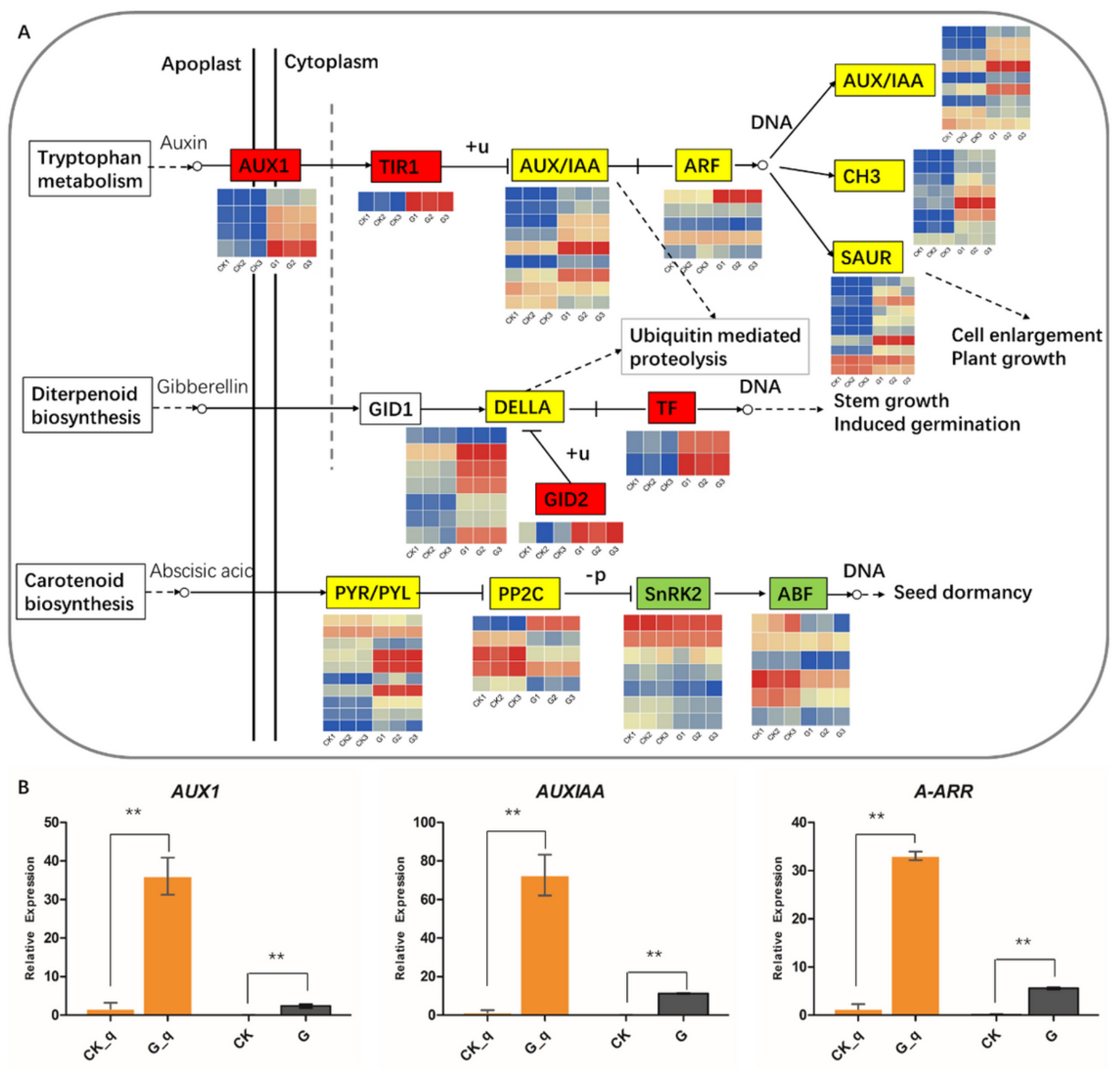

Figure 6

The DEGs expression enriched in auxin, gibberellin (GA) and abscisic acid (ABA) biosynthesis pathway (A), expression levels are indicated by the heatmap at $\mathrm{G}$ and CK group, estimated using log2(fold change) for each transcript. Genes marked with red indicate up-regulated, green was down-regulated, yellow has both up- and down-regulated genes in germinated seeds. The genes in those pathway determined by qRTPCR (B). In the histogram, CK_q and G_q represent qPCR analysis; CK and G represent the FPKM value of RNA_seq (**p<0.01). 


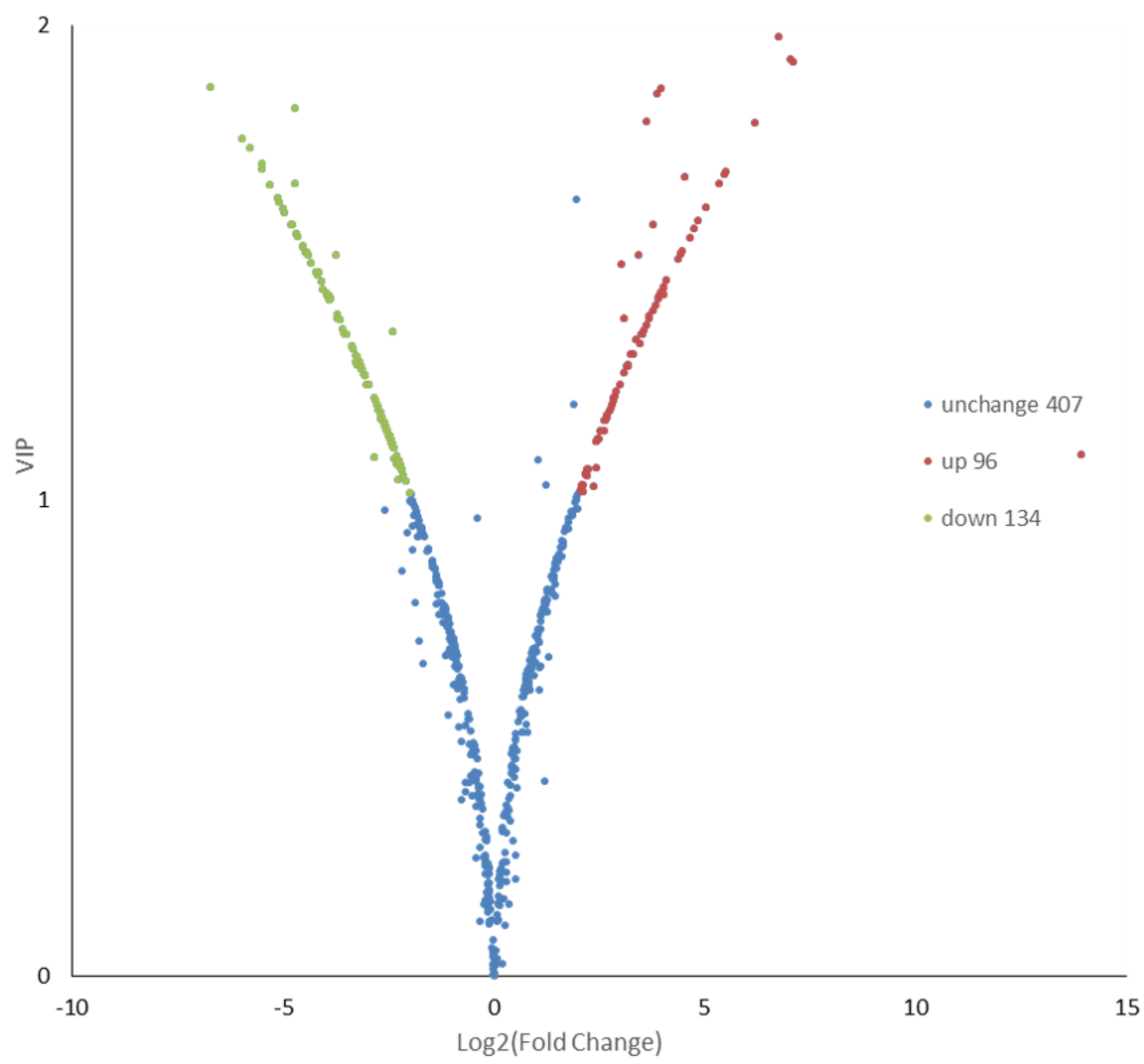

Figure 7

Volcano plots of differentially metabolites between germinated and control seeds in Polygonatum cyrtonema Hua. 
A

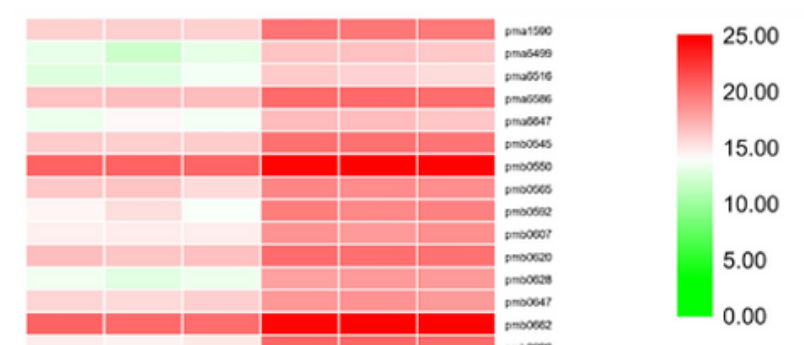

C

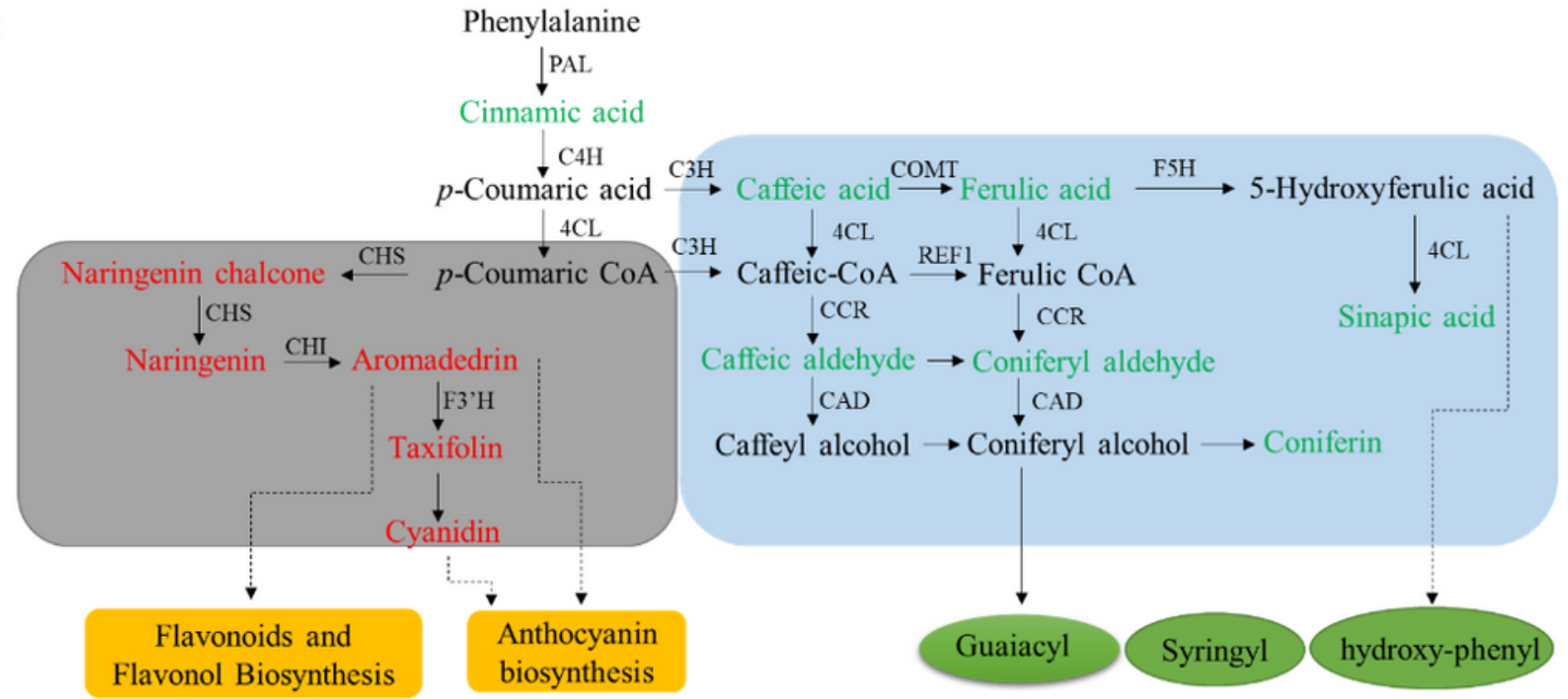

\section{Figure 8}

The differentially metabolites of flavonoids $(A)$ and hydroxycinnamyl $(B)$ between germinated and control seeds in Polygonatum cyrtonema Hua. Accumulation of differential metabolites in flavonoid biosynthesis and phenylpropanoid metabolism pathway in germinated vs CK seeds (C). Metabolites marked with red indicate up-regulated, green has down-regulated in germinated seeds. 


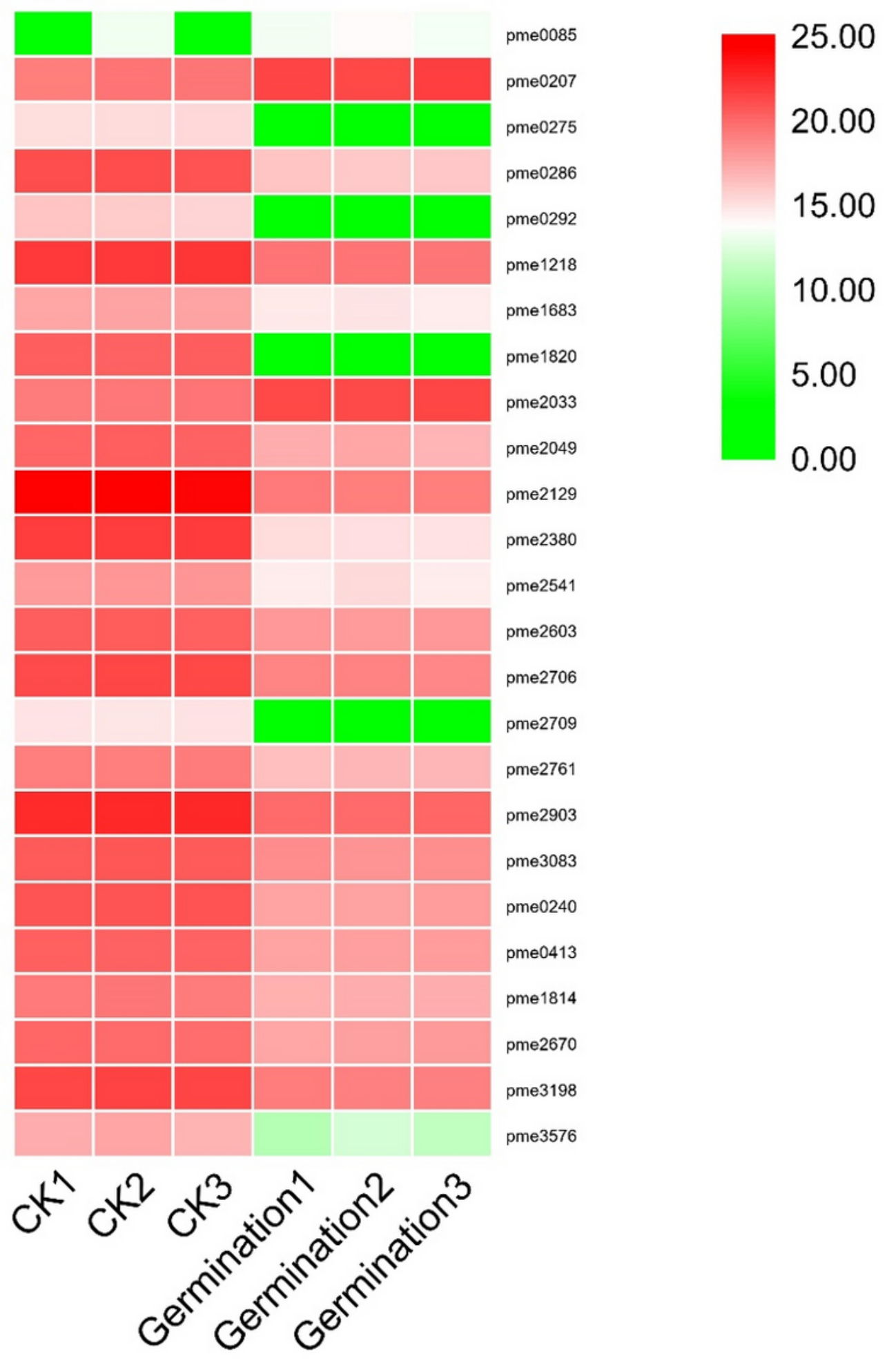

\section{Figure 9}

The differentially metabolites of organic acid between germinated and control seeds in Polygonatum cyrtonema Hua.

\section{Supplementary Files}


This is a list of supplementary files associated with this preprint. Click to download.

- Supplementalfile4.xlsx

- Supplementalfile3.xlsx

- TableS1.xlsx

- Supplementalfile7.xlsx

- Supplementalfile1.xIsx

- Supplementalfile6.xlsx

- Supplementalfile5.xlsx

- Supplementalfile2.xIsx 